\title{
Understanding of variability properties in very low mass stars and brown dwarfs
}

\author{
Soumen Mondal ${ }^{1}$, Samrat Ghosh ${ }^{1}$, Dhrimadri Khata ${ }^{1}$, Santosh Joshi ${ }^{2}$, \\ Ramkrishna Das ${ }^{1}$
}

\author{
${ }^{1}$ S. N. Bose National Centre for Basic Sciences, JD Block, Salt Lake, Kolkata-700106, India \\ ${ }^{2}$ Aryabhatta Research Institute of Observational Sciences (ARIES), Manora Peak, Nainital, India
}

\begin{abstract}
We report on photometric variability studies of a L3.5 brown dwarf 2MASS J00361617+1821104 $(2 \mathrm{M} 0036+18)$ in the field and of four young brown dwarfs in the star-forming region IC 348. From muti-epoch observations, we found significant periodic variability in $2 \mathrm{M} 0036+18$ with a period of $2.66 \pm 0.55$ hours on one occasion while it seemed to be non-variable on three other occasions. An evolving dust cloud might cause such a scenario. Among four young brown dwarfs of IC 348 in the spectral range M7.25-M8, one brown dwarf 2MASS J03443921+3208138 shows significant variability. The K-band spectra $(2.0-2.4 \mu \mathrm{m})$ of nine very low mass stars (M1-M9 V) are used to characterize the water band index $\left(\mathrm{H}_{2} \mathrm{O}-\mathrm{K} 2\right)$. We found that it is strongly correlated with the surface temperature of $\mathrm{M}$ dwarfs.
\end{abstract}

\section{Introduction}

The term "very low mass star" (VLMs) refers to a main sequence star with a spectral type from mid-K to late-M and a mass from about $0.6 \mathrm{M}_{\odot}$ down to the brown dwarf limit of $0.075 \mathrm{M}_{\odot}$ (Allard et al. 1997). The metallicities of VLMs in the solar neighborhood span up to $\pm 0.5-1.0$ dex around the solar value. However, the metallicity in these dwarfs shows a wide-range from metal-rich in the galactic disk and open clusters (e.g. Pleiades, Hyades, Ophiuchus, Persei) to metal-poor dwarfs of the galactic halo and globular clusters (Allard et al. 1997). Brown dwarfs (BDs) are sub-stellar objects with a mass below $0.075 \mathrm{M}_{\odot}$ and a spectral type L, T, or Y (Kirkpatrick 2005). They don't have enough mass to fuse the hydrogen inside the core. They burn deuterium instead, as the threshold energy for deuterium fusion is lower compared to the one of hydrogen fusion. Theoretical models are found challenging to explain the spectral features of these dwarfs (e.g., Allard et al. 2001; Marley et al. 2002; Helling et al. 2008).

Clouds are particularly unique and valuable features of the atmospheres of late-M, L, and earlyT type dwarfs as photospheric conditions are conducive to condensate several minerals and metals. The raining out of clouds happens in later T dwarfs (Tsuji et al. 1996; Lodders 2002; Helling et al. 2008; Marley et al. 2010). Rotational modulation due to an inhomogeneous cloud coverage is always suggested as the probable cause of the photometric variability as seen in these dwarfs (Bailer-Jones \& Mundt 1999; Bailer-Jones \& Mundt 2001; Gelino et al. 2002; Koen 2006; Maiti 2007; Lane 2007; Khandarika et al. 2013; Radigan et al. 2014; Metchev et al. 2015). BDs are rapid rotators with 
Table 1: Log of photometric and spectroscopic observations. For each night, we list the observed targets, the telescope/instrument and type of observation/filter combination used, the exposure time in seconds and the total number of frames.

\begin{tabular}{|l|l|l|l|l|l|}
\hline Date & Target & Telescope/Instrument & Type/Filters & Texp & Frames \\
\hline 06 Dec 2007 & 2M0036+18 (L3.5 V) & $1.04 \mathrm{~m}$ ST/CCD & Photometry/I & 200s & 35 \\
02 Jan 2008 & 2M0036+18 & $1.04 \mathrm{~m}$ ST/CCD & Photometry/I & $400 \mathrm{~s}$ & 20 \\
25 Sep 2008 & 2M0036+18 & $1.04 \mathrm{~m}$ ST/CCD & Photometry/I & $300 \mathrm{~s}$ & 20 \\
01 Nov 2010 & 2M0036+18 & $1.04 \mathrm{~m}$ ST/CCD & Photometry/I & $300 \mathrm{~s}$ & 30 \\
18 Dec 2016 & IC 348 BDs (M7.25-M8 V) & $1.3 \mathrm{~m}$ DFOT/CCD & Photometry/I & $400 \mathrm{~s}$ & 28 \\
20 Dec 2016 & IC 348 BDs & $1.3 \mathrm{~m}$ DFOT/CCD & Photometry/I & $400 \mathrm{~s}$ & 43 \\
17 Feb 2016 & GJ3395 (M1 V) & 2m HCT/TIRSPEC & Spectroscopy/HK & $500 \mathrm{~s}$ & 2 \\
17 Feb 2016 & HD95735 (M1 V) & 2m HCT/TIRSPEC & Spectroscopy/HK & $100 \mathrm{~s}$ & 2 \\
22 Dec 2016 & GJ48 (M3 V) & 2m HCT/TIRSPEC & Spectroscopy/HK & $100 \mathrm{~s}$ & 2 \\
22 Dec 2016 & GJ9949 (M4 V) & 2m HCT/TIRSPEC & Spectroscopy/HK & $100 \mathrm{~s}$ & 2 \\
\hline
\end{tabular}

rotation periods of the order of hours (Zapatero Osorio et al. 2006). Their photometric variability can be observed within a few nights of photometric monitoring using small to moderate-sized telescopes. Another characteristic of the photometric variability in these class of objects is the lack of stability in both the periodicity and amplitude, which might be due to the evolving cloud features. To provide a comprehensive understanding of the variability of VLMs and BDs in the field as well as in young star-forming regions, we have started an observational program in the optical/near-infrared (NIR) wavelength region.

Unfortunately, the variability of the amplitudes of BDs is quite small (less than $100 \mathrm{mmag}$ ), and hence the variability detection is quite challenging. Thus, it is important to explore or quantify the dependence of variability on other parameters such as rotation, temperature, surface gravity, metallicity, or the overall cloud content for such dwarfs. In this paper, we present a sample of 5 BDs for variability studies, one in the field and four young BDs in the star-forming region IC 348. The NIR K-band $(2-2.4 \mu \mathrm{m})$ spectroscopic observations on $\mathrm{M}$ dwarfs are presented here to understand the cloud properties using the water bands. In Section 2, we describe our observations including the instruments, the data reduction procedures, and the spectroscopic and photometric analysis. In Section 3 , we present the light curves for our sources and the results derived from them. We also describe the analysis of NIR spectroscopic observations of M dwarfs. Finally, we summarize our results in Section 4.

\section{Observations and data reduction}

\subsection{Optical photometric observations}

The time-series photometric observations of our sample were obtained using the 1.3-m Devasthal Fast Optical Telescope (DFOT; Devathal observatory, Nainital, India) and the 1.04-m Sampuranand Telescope (ST; ARIES campus, Nainital, India). Optical CCD imaging data from the ST were obtained with the standard $2 \mathrm{~K} \times 2 \mathrm{~K}$ CCD camera. The $\mathrm{f} / 13$ optics of the $\mathrm{ST}$ provides a pixel scale of about 0.37 arcsec for $24 \mu \mathrm{m}$ size pixels and a field of view of about $13 \times 13 \operatorname{arcmin}^{2}$. The DFOT was equipped with a $13.5-\mu \mathrm{m}$ pixel size, $2 \mathrm{~K} \times 2 \mathrm{~K}$ Andor camera, which covers a square area of about 18 arcmin sky on a side with a pixel scale of $0.53 \operatorname{arcsec}$ (Sagar et al. 2011). The log of our photometric observations is given in Table 1. 
CCD raw images are processed using the standard reduction package $I R A F^{1}$. The image reduction follows the standard CCD-reduction procedures like bias-subtraction, dark-subtraction and flat-field correction including cosmic rays removal with the CCDPROC task within IRAF. For each processed image, we performed aperture photometry using the APPHOT task of IRAF for all the sources on the CCD frames. The aperture sizes were chosen 3 to 4 times the FWHM of the stars, which corresponds to $6-7$ arcsec on the sky. Differential photometry was performed on all images to get a better precision for the light curves by removing the effects of atmospheric transparency and other instrumental effects. In differential photometry, an average light curve for each night is created for as many as possible non-variable reference stars available in the frame for a particular run. Non-variable reference stars in the frames should have a relatively low root-mean-square (RMS) in the light curves, should be isolated on the frame, and should have a brightness similar to the one of the target.

\subsection{Near-infrared spectroscopic observations}

The NIR spectroscopic observations for a sample of a few M dwarfs were conducted with the Tata Institute of Fundamental Research (TIFR) Near-Infrared Spectrometer and Imager (TIRSPEC) attached to the 2-m Himalayan Chandra Telescope (HCT) in Hanle (India). It has an 1024×1024 Hawaii-1 array detector and a spectral resolution of about 1200. The wavelength coverage of TIRSPEC spectra is $1.02-1.20 \mu \mathrm{m}$ for order $\mathrm{Y}, 1.21-1.48 \mu \mathrm{m}$ for order $\mathrm{J}, 1.49-1.78 \mu \mathrm{m}$ for order $\mathrm{H}$, and $2.00-2.35 \mu \mathrm{m}$ for order K. Details of the TIRSPEC instrument can be found in Ninan et al. (2014). We have taken the HK $(1.49-2.35 \mu \mathrm{m})$ spectra on M dwarfs in the cross-disperser mode of TIRSPEC. The spectra were taken at two different positions along the slit immediately after each other to be able to subtract the sky. Multiple frames were taken to improve the signal-to-noise ratio (SNR).

The spectroscopic analysis was done using the APALL task of IRAF. The TIRSPEC data were also reduced with the TIRSPEC pipe-line ${ }^{2}$ (Ninan et al. 2014) and cross-checked with the IRAF reduction. Both techniques agree well. The wavelength calibration in TIRSPEC data was done using the Argon lamp of the calibration unit. The strong absorption of $\mathrm{CO}_{2}$ and $\mathrm{H}_{2} \mathrm{O}$ bands in the Earth's atmosphere is also recorded in the spectra. To remove these telluric features, we divided the spectra of our targets by corresponding spectra of a telluric standard star. This procedure is followed by the flux calibration of the spectra of the target stars using the spectrum of a standard star.

\subsection{Period determination}

We used the Lomb-Scargle (LS) periodogram (Lomb 1976; Scargle 1982) to determine the most likely period in the light curves of BDs. The LS method computes the Fourier power over an ensemble of frequencies and is able to find significant periodicities even for unevenly sampled data. We used the LS algorithm taken from the publicly available starlink ${ }^{3}$ software database. The periods were further verified with the software Period0 $4^{4}$ (Lenz \& Breger 2005) and an online available web-based ${ }^{5}$ LS method. This software was used successfully for period analysis in any unevenly sampled photometric data set (Mondal et al. 2010). The periodograms were searched for significant peaks to detect periodic variability. When a significant peak was found, the data were phase folded to this period. Next, those phase folded light curves were subjected to a careful visual inspection to get rid of spurious periods. The different algorithms were used to converge to the final value of the period for an individual night as well as for the combined dataset.

\footnotetext{
${ }^{1}$ Image Reduction and Analysis Facility

${ }^{2}$ https://github.com/indiajoe/TIRSPEC/wiki

${ }^{3} \mathrm{http}: / /$ starlink.jach.hawaii.edu/starlink

${ }^{4} \mathrm{http}: / /$ www.univie.ac.at/tops/Period04

${ }^{5} \mathrm{http}$ ://exoplanetarchive.ipac.caltech.edu/docs/tools.html
} 


\section{Results and discussion}

\subsection{Photometric variability of brown dwarfs}

\subsubsection{The L3.5 field brown dwarf 2MASS J00361617+1821104 (2M0036+18)}

2M0036+18 is an L3.5 BD (Reid et al. 2000). The object is located at distance of $8.77 \pm 0.06 \mathrm{pc}$. The bolometric luminosity is estimated as $\mathrm{L}_{b o l} \sim 10^{-3.97} \mathrm{~L}_{\odot}$, the radius as $0.09 \pm 0.01 \mathrm{R}_{\odot}$, and the effective temperature lies in the range 1700-1950 K (Dahn et al. 2002; Leggett et al. 2002). The mass of the object is estimated to be $0.076 \mathrm{M}_{\odot}$ from the surface gravity $\log g \approx 5.4$ (Schweitzer et al. 2001). $2 \mathrm{M} 0036+18$ was detected at radio wavelengths with a quiescent flux of $259 \pm 19 \mu \mathrm{Jy}$ and $134 \pm 16 \mu \mathrm{Jy}$ at 4.9 and $8.5 \mathrm{GHz}$, respectively (Berger et al. 2002, 2005). A periodicity of 3 hours in 2M0036+18 was first reported from radio observations (Berger et al. 2002). Later, a similar period was also recovered from the I-band observations in the optical (Lane et al. 2007).

We have gathered a time-series of I-band observations of 2M0036+18 from 06 December 2007 to 1 November 2010 ( $\sim 3$ yrs.). A log of our observations is given in Table 1 . The differential light curves observed at four epochs are shown in Figs. 1 and 2. Notably, only on 6 December 2007, the light curve showed a significant variability, while the source was non-variable at the other epochs. Period analysis was performed using the LS method as described in Section 2.3. We found a significant period of $2.66 \pm 0.55$ hours for the light curve observed on 6 December 2007. The periodogram and phase-folded light curve is shown in Fig. 3. Our estimated period agrees well with the earlier reported period values (Berger et al. 2002; Lane 2007).

The optical photometric variability of $2 \mathrm{M} 0036+18$ is a compelling case. The source was monitored first in the I-band by Gelino et al. (2002) who concluded that it was non-variable with an upper-limit RMS amplitude of 9 mmag. Based on simultaneous radio observations at 4.9 and 8.5 $\mathrm{GHz}$, Berger et al. (2002) first reported a periodic radio emission ( $\mathrm{P} \sim 3$ hours). Further simultaneous observations in radio, $\mathrm{X}$-ray, and $\mathrm{H} \alpha$ showed the absence of $\mathrm{X}$-ray and $\mathrm{H} \alpha$ emission but the presence of persistent radio emission over a timescale of $\sim 3$ yrs (Berger et al. 2005). It suggested that a magnetic field of about 200 Gauss could be responsible for such unexpected radio emission from a cool BD (Berger et al. 2005). Subsequently, Maity (2007) monitored the source in the Rand I-bands over several epochs from 19 September 2004 to 29 October 2005. They found significant variability on a few occasions while it was non-variable at most times. Lane (2007) monitored the source in the I-band on 19 September 2006 and found that the source was significantly variable with a rotational modulation period of about 3 hours. Koen (2013) monitored the source in the Rand I-bands on 08-09 September 2005 and found variability in the I-band, but no variability in the contemporaneous observations in the R-band. All previous observations reveal non-persistent optical variability for $2 \mathrm{M} 0036+18$. We got a similar result from our three years optical monitoring program. Such optical variability might be caused by a scenario of formation and dissipation of cloud features as predicted by several theoretical models and observed on a few occasions for other BDs.

\subsubsection{Young brown dwarfs in IC 348}

IC 348 is a nearby ( $\sim 310 \mathrm{pc})$ and young (2-3 Myr) star-forming region in the Perseus molecular cloud. Membership of IC 348 is very well characterized by several authors (Luhman et al. 2003; Luhman et al. 2005; Muench et al. 2007). Spectral types of VLMs and BDs were estimated using NIR spectroscopy (Luhman et al. 2005).

Based on brightness observations in the I-band with a reasonable photometric accuracy, we have selected four BDs in the spectral range M7.25 - M8 with an I-band brightness between 17 and $18.6 \mathrm{mag}$ inside the field of view of IC 348: 2MASS J03443920+3208136 (M8; I = $18.17 \mathrm{mag}$ ), 

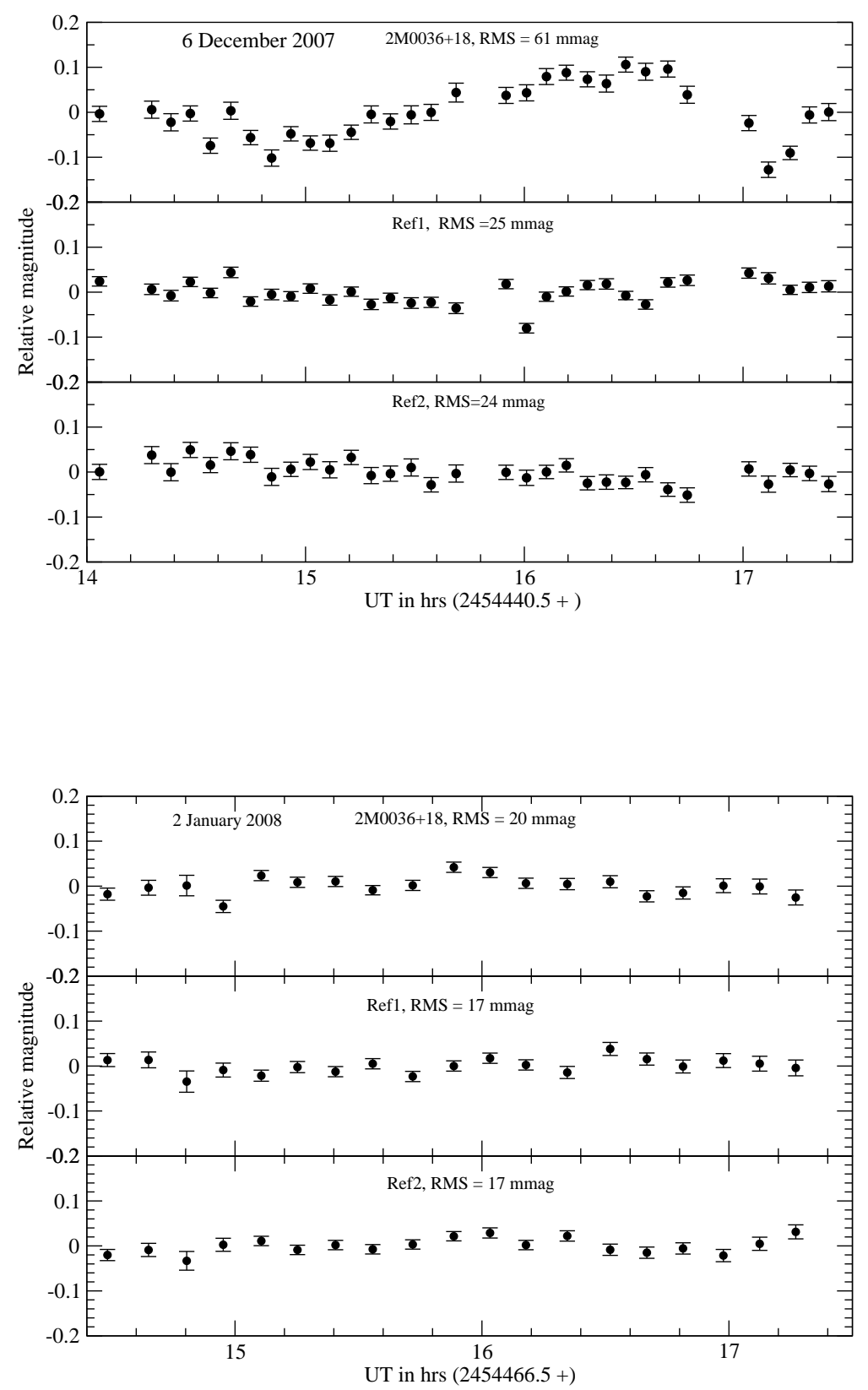

Figure 1: Differential photometry light curves in the I-band observed on 6 December 2007 (top) and 2 January 2008 (bottom). In each panel, the upper light curve is observed for 2M0036+18 while the middle and lower light curves are those of reference stars of similar brightness in the observed field. 

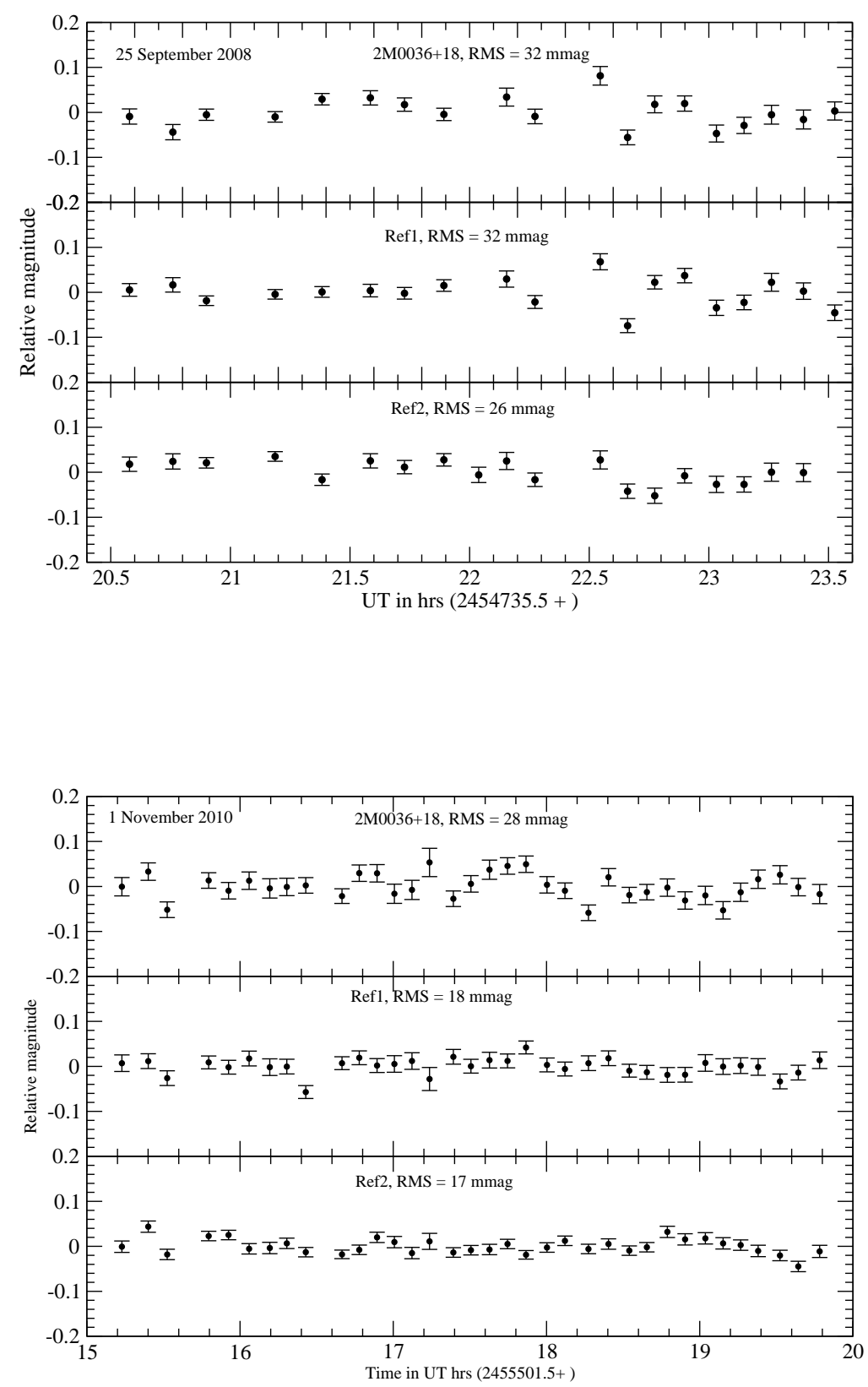

Figure 2: Same as Fig. 1 but for the differential photometry light curve in the I-band observed on 25 September 2008 (top) and 01 November 2010 (bottom). 

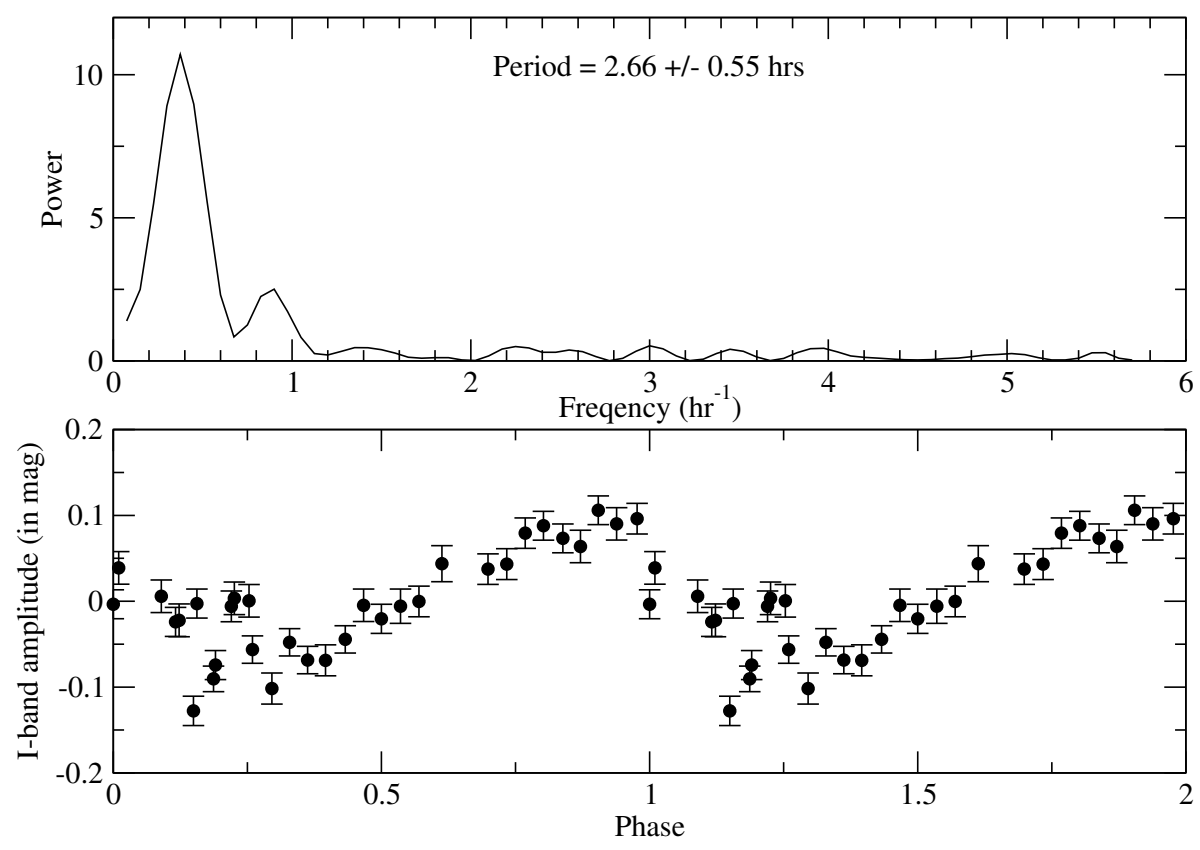

Figure 3: The periodiogram using the Lomb-Scargle method (top) and the phased light curve using a period of 2.66 hours and the reference epoch JD 2454441.31493 (bottom) of the observations in the I-band of 2M0036+18 obtained on 6 December 2007.

2MASS J03435638+3209591 (M7.25; I = $18.61 \mathrm{mag})$, 2MASS J03441558+3209218 (M7.5; I = $17.64 \mathrm{mag}$ ), and SSTc2d J034434.1+320657 (M7.25; I = $17.09 \mathrm{mag})$. Details of the observations are listed in Table 1. The light curves of these four BDs are shown in Figs. 4 and 5. From the differential photometric light curve analysis, we have found that the observed variations of 2MASS $\mathrm{J} 03443920+3208136$ are significant $\left(\sigma_{r m s} / \sigma_{\text {ref }} \approx 2.4 ; \sigma_{r m s} / \sigma_{\text {errormag }} \approx 1.9\right)$. The observations of 2MASS J03435638+3209591 show evidence for variability $\left(\sigma_{r m s} / \sigma_{r e f} \geq 3.3\right)$, but the large errors on the individual magnitudes ( $\sigma_{\text {errormag }} \approx 47$ and $74 \mathrm{mmag}$ ) hamper to be conclusive. For 2 MASS $\mathrm{J} 03441558+3209218$ and SSTc2d J034434.1+320657, there is no evidence for variability with an amplitude above $\sim 30 \mathrm{mmag}$ and $\sim 20 \mathrm{mmag}$, respectively. A large aperture telescope, like the 3.6-m Devasthal Optical Telescope (DOT) of the Aryabhatta Research Institute of Observational Sciences (ARIES), will help to improve the characterization of the variability of these faint BDs.

\subsection{Near-IR spectroscopic studies of selected low mass M-dwarfs}

We have studied the basic physical properties of nine VLM M dwarfs (M1V-M9 V) using NIR K-band spectra $(2-2.4 \mu \mathrm{m})$. Four spectra for M dwarfs (M1-M4 V) were taken using the TIRSPEC instrument on the HCT. We also used six archival spectra of M5-M9 V stars available from the SPEX IRTF Library, which were collected using the SpeX spectrograph on the 3-m NASA Infrared Telescope Facility (IRTF) (Rayner et al. 2009).

By studying several atomic and molecular indices, we aim to quantify the changes in the strength of these absorption features with spectral type. We are exploring the correlation with different fundamental parameters (like the effective temperature $T_{\text {eff }}$, the surface gravity $\log g$, and the metallicity $[\mathrm{M} / \mathrm{H}])$ of $\mathrm{M}$ dwarfs. Complementary to these observations, synthetic spectra from the BT-SETTL version of the PHOENIX atmosphere model grids (Allard et al. 2012) were used to support a qualitative validation of our approach.

The NIR spectra of M dwarfs are dominated by features of the broad absorption-band of $\mathrm{H}_{2} \mathrm{O}$. Their strength is known to correlate strongly with $T_{\text {eff }}$ and spectral type. Using these features, several 
18 December, 2016
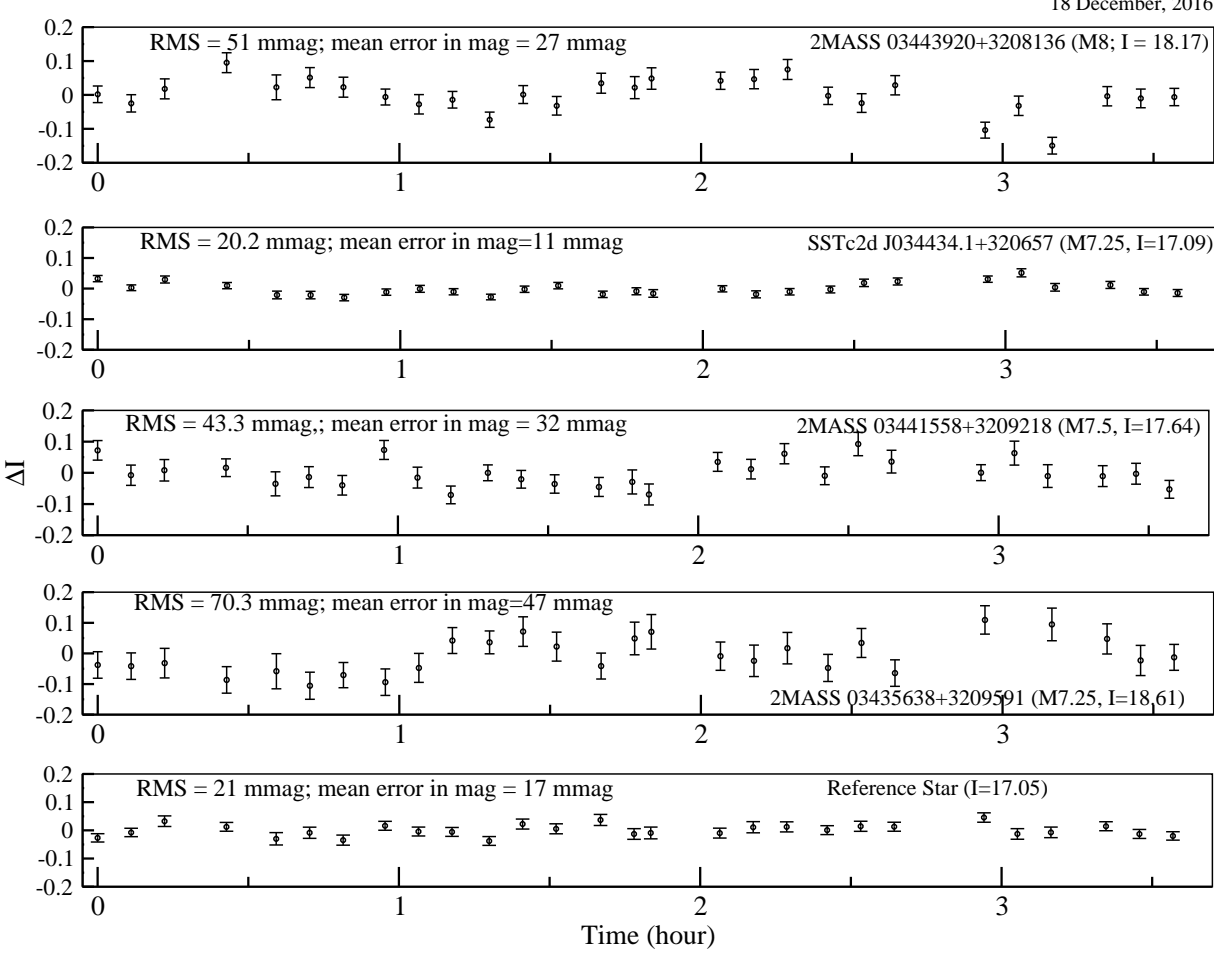

Figure 4: The I-band light curves of four young BDs in IC 348 (four top panels) and of a reference star of similar brightness on the CCD frames (bottom panel) as observed on 18 December 2016.

20 December, 2016
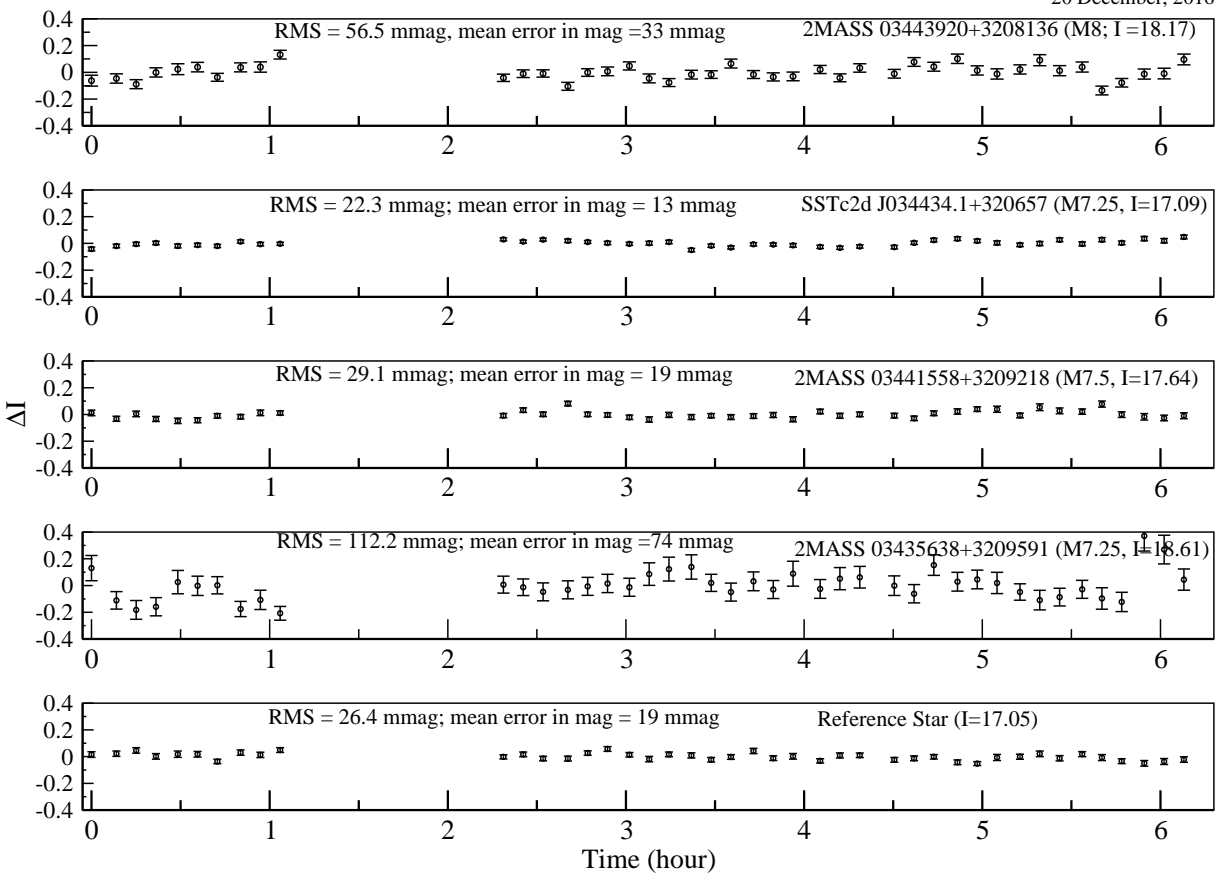

Figure 5: Same as Fig. 4 but for I-band observations obtained on 20 December 2016. 


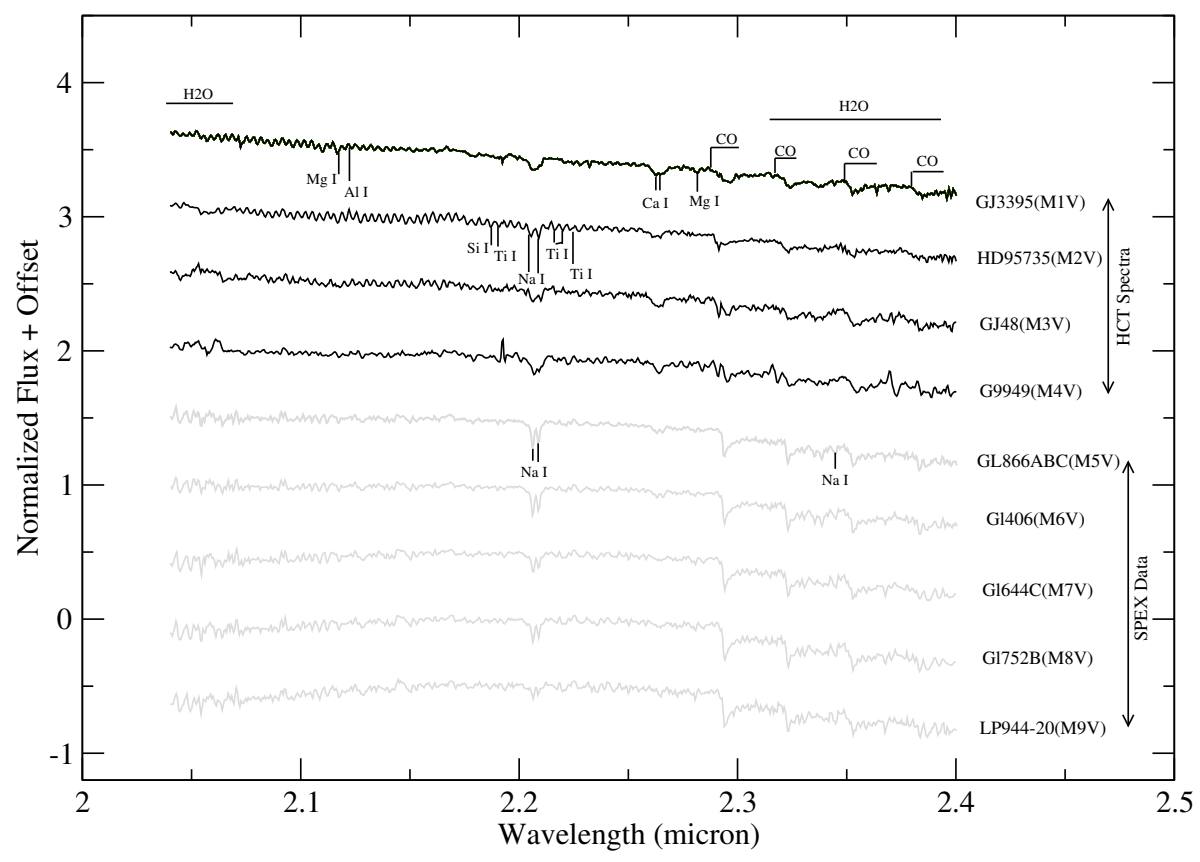

Figure 6: K-band spectra (2.0-2.4 $\mu \mathrm{m})$ that we obtained for four VLMs (M1-M9 V) with the TIRSPEC instrument of the HCT (black) and archival spectra of the SPEX IRTF library (Rayner et al. 2009; grey). Several atomic and molecular features are marked, which show changes with spectral type.

authors have successfully used the $\mathrm{H}_{2} \mathrm{O}$-based indices to correlate spectral type and $T_{\text {eff }}$, and established the empirical calibration relations for the K-band $\mathrm{Na}$ I doublet and the $\mathrm{Ca}$ I triplet along with $\left(\mathrm{H}_{2} \mathrm{O}-\mathrm{K}\right)$ absorption indices (Rojas-Ayala et al. 2012; Newton et al. 2015). Following Rojas-Ayala et al. (2012), we estimate here the $\left(\mathrm{H}_{2} \mathrm{O}-\mathrm{K} 2\right)$ index of $\mathrm{M}$ dwarfs to understand any correlation with the cloud properties in VLMs and BDs.

In Fig. 6, the K-band spectra of nine VLMs (M1-M9V) are shown. Several atomic and molecular features are marked, which show changes with spectral type. In the upper panel of Fig. 7, the $\left(\mathrm{H}_{2} \mathrm{O}-\right.$ K2) indices for different metallicities are shown as a function of model $T_{\text {eff }}$, which are estimated from the PHOENIX BT-SETTL synthetic spectra developed by Allard et al. (2012). The observed $\left(\mathrm{H}_{2} \mathrm{O}-\right.$ $\mathrm{K} 2$ ) indices from K-band spectra of $\mathrm{M}$ dwarfs are over-plotted on that graph. In the lower panel of Fig. 7, the observed $\left(\mathrm{H}_{2} \mathrm{O}-\mathrm{K} 2\right)$ indices versus the spectral sub-types of $\mathrm{M}$ dwarfs are shown together with a linear fit between the spectral type and the $\left(\mathrm{H}_{2} \mathrm{O}-\mathrm{K} 2\right)$ index. We found that the $\left(\mathrm{H}_{2} \mathrm{O}-\mathrm{K} 2\right)$ index is strongly correlated with the surface temperature of the star. Furthermore, the dependence on metallicity is not as strong as predicted by the PHOENIX BT-SETTL synthetic spectral models developed by Allard et al. (2012).

\section{Summary}

We studied the photometric variability of a L3.5 BD in the field and of four young (2-3 Myr) M7.25M8 BDs in the star-forming region IC 348. From muti-epoch observations, we found significant periodic variability in the data of the field star $2 \mathrm{M} 0036+18$ with a period of $2.66 \pm 0.55$ hours on one occasion, but at the three other epochs it seems to be non-variable. Evolving dust clouds might be a probable cause for such a scenario. Among the four studied BDs of IC 348, one shows significant variability (2MASS J03443921+3208138). Another one (2MASS J03441558+3209219) also seems to be variable but the large errors on the individual magnitudes prevent a safe conclusion.

From NIR spectroscopy observations in K-band for a sample of M dwarfs, we have characterized 

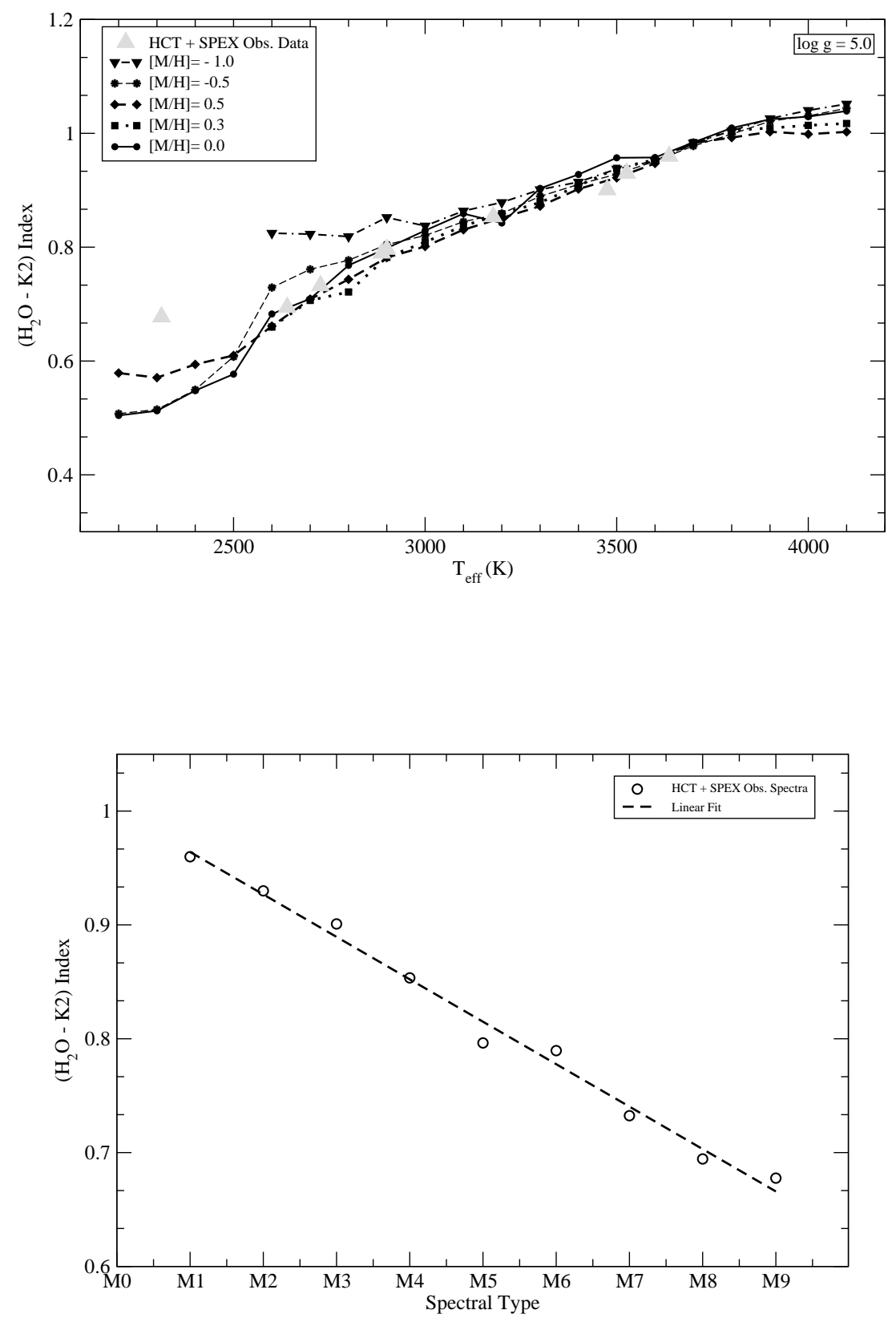

Figure 7: (top) The $\left(\mathrm{H}_{2} \mathrm{O}-\mathrm{K} 2\right)$ index for different metallicities estimated from the PHOENIX BTSETTL synthetic spectra developed by Allard et al. (2012) as a function of model $T_{\text {eff }}$. The observed $\left(\mathrm{H}_{2} \mathrm{O}-\mathrm{K} 2\right.$ ) indices from K-band spectra of $\mathrm{M}$ dwarfs from TIRSPEC and SPEX are overplotted (grey triangles). (bottom) The observed $\left(\mathrm{H}_{2} \mathrm{O}-\mathrm{K} 2\right)$ indices as a function of spectral type. The observed indices are given with black circles while the dashed line is a linear fit between them. 
the water band $\left(\mathrm{H}_{2} \mathrm{O}-\mathrm{K} 2\right)$ index to understand their cloud properties. We found that the $\left(\mathrm{H}_{2} \mathrm{O}-\mathrm{K} 2\right)$ index is strongly correlated with the surface temperature of the star. Furthermore, the dependence on metallicity is not as strong as predicted by the PHONEIX BT-SETTL synthetic spectral models developed by Allard et al. (2012).

\section{Acknowledgements}

This research work is supported by the S N Bose National Centre for Basic Sciences under the Department of Science and Technology, Govt. of India. The authors are thankful to the JTAC members and staff of the 1.3-m Devasthal optical telescope operated by Aryabhatta Research Institute of Observational Sciences (Nainital), the HTAC members and the staff of the HCT, operated by Indian Institute of Astrophysics (Bangalore).

\section{References}

Allard F., Hauschildt P. H., Alexander D. R., Starrfield S. 1997 ARA\&A, 35, 137

Allard F., Hauschildt P. H., Alexander D. R., Tamanai A., Schweitzer A. 2001, APJ, 556, 357

Allard F., Homeier D., Freytag B. 2012, RSPTA, 370, 2765

Bailer-Jones C. A. L., Mundt R. 1999, A\&A, 348, 800

Bailer-Jones C. A. L., Mundt R. 2001, A\&A, 367, 218

Berger E. 2002, ApJ, 572, 503

Berger E., Rutledge R. E., Reid I. N. et al. 2005, APJ, 627, 960

Dahn C. C, Harris H. C., Vrba F. J. et al., 2002, AJ, 124, 1170

Gelino C. R., Marley M. S., Holtzman J. A., Ackerman A. S., Lodders K. 2002, APJ, 577, 433

Helling C., Ackerman A., Allard F. et al. 2008, MNRAS, 391, 1854

Khandrika H., Burgasser A. J., Melis C. et al. 2013, AJ, 145, 71

Kirkpatrick J. D. 2005, ARA\&A, 43, 195

Koen C. 2006, MNRAS, 367, 1735

Koen C. 2013, MNRAS, 428, 2824

Lane C. 2007, ApJ, 668L, 163

Leggett S. K., Golimowski D. A., Fan X. et al. 2002, ApJ, 564, 452

Lenz P., Breger M. 2005, CoAst, 146, 53

Lodders K. 2002, ApJ, 577, 974

Lomb N. R. 1976, AP\&SS, 39, 447

Luhman K. L., Lada E. A., Muench A. A., Elston A. J. 2005, APJ, 618, 810

Luhman K. L., Stauffer J. R., Muench A. A. et al. 2003, ApJ, 593, 1093

Maiti M. 2007, AJ, 133, 1633

Marley M. S., Saumon D., Goldblatt C. 2010, APJ, 723, 117

Marley M. S., Seager S., Saumon D. et al. 2002, APJ, 568, 335

Metchev S. A., Heinze A., Apai D., et al. 2015, ApJ, 799, 154

Mondal S., Lin C. C., Chen W. P. et al. 2010, AJ, 139, 2026

Muench A. A., Lada C. J., Luhman K. L., Muzerolle J., Young E. 2007, AJ, 134, 411

Newton E. R., Charbonneau D., Irwin J., Mann A. W. 2015, APJ, 800, 85

Ninan J. P., Ojha D. K., Ghosh S. K. et al. 2014, JAI, 3, 1450006

Radigan J., Lafrenire D., Jayawardhana R., Artigau E. 2014, ApJ, 793, 75

Rayner J. T., Cushing M. C., Vacca W. D. 2009, ApJS, 185, 289

Reid I. N., Kirkpatrick J. D., Gizis J. E. et al. 2000, AJ, 119, 369.

Rojas-Ayala B., Covey K. R., Muirhead P. S., Lloyd J. P. 2012, ApJ, 748, 93

Sagar R., Omar A., Kumar B. et al. 2011, CSci, 101, 1020

Scargle J. D. 1982, APJ, 263, 835

Schweitzer A., Gizis J. E., Hauschildt P. H., Allard F., Reid I. N. 2001, ApJ, 555, 368

Tsuji T., Ohnaka K., Aoki W. 1996, A\&A, 305, 1

Zapatero Osorio M. R., Martín E. L., Bouy H. et al. 2006, APJ, 647, 1405 